\title{
RESEARCH NOTES \\ A NEW PROOF OF A THEOREM OF TOEPLITZ IN SUMMABILITY THEORY
}

\section{P. ANTOSIK and V.K. SRINIVASAN}

Department of Mathematics

University of Texas at E1 Paso

E1 Paso, Texas 79968

(Received January 24, 1985 and in revised form June 11, 1985)

ABSTRACT. The object of this paper is to give a new proof of a Theorem in Summability, as an application of a result of Antosik.

KEY WORDS AND PHRASES. Summability.

1980 AMS SUBJECT CLASSIFICATION CODE. $40 \mathrm{C}$.

1. INTRODUCTION.

From time to time as general concepts develop, new proofs of old theorems are made possible. The so called theorem of Toeplitz in summability theory gives necessary and sufficient conditions for an infinite matrix $A=\left(a_{m, n}\right)$ of real (or complex numbers) numbers to be regular. The first proof, entirely analytical is given in Hardy [1]. The second proof is obtained by using the uniform boundedness principle of Functional analysis. In this paper in section 2, we obtain a new proof of Toeplitz's theorem based on a result of Antosik given in his paper [2]. The above method has other applications also.

2. MAIN RESULTS.

Let $A=\left(a_{m, n}\right)$ be an infinite matrix of real numbers. Given a sequence [s $\left.{ }_{n}\right]$ of real numbers if

$$
t_{m}=\sum_{n=1}^{\infty} a_{m, n} s_{n}
$$

exists for each $m$ and if

$$
\underset{m \rightarrow \infty}{11 m} t_{m}=L
$$

exists, then $L$ is called the $A\left(\underset{n \rightarrow \infty}{\lim } s_{n}\right)$ and we write

$$
A\left(\lim _{n \rightarrow \infty} s_{n}\right)=L
$$

We also say that $\left\{s_{n}\right\}$ is A-summable to $L$ if (2.3) holds.

Given $A=\left(a_{m, n}\right)$ let $A^{*}$ be the linear space of all A-summable sequences. If (c) denotes the space of convergent sequences, then $A$ is called a convergence preserving transformation if $(c) \subseteq A^{*}$. A is called a regular transformation if $A$ is convergence preserving and in addition for each convergent sequence [ $s_{n}$ ]

$$
A\left(\lim _{n \rightarrow \infty} s_{n}\right)=\lim _{n \rightarrow \infty} s_{n}
$$


We now state the diagonal theorem of Antosik [2] used in this paper.

THEOREM A (Diagonal Theorem for Non-negative Matrices). Let $A=\left(x_{1, j}\right)$ be a matrix of non-negative real numbers such that

$$
\begin{aligned}
& \frac{1+m}{j \neq \infty}\left(x_{1, j}\right)=0(\text { for all } i \in N) \\
& \left.\underset{1 \neq \infty}{1 \neq \infty}\left(x_{i, j}\right)=0 \text { (for all } j \in N\right) \\
& \lim _{i \neq \infty}\left(x_{i, i}\right)=0
\end{aligned}
$$

then there exists an increasing sequence of positive integers $\left\{\mathrm{p}_{1}\right\}$ such that

$$
\sum_{i, j} x_{p_{i}, p_{j}}<\infty
$$

$N$ being the set of natural numbers. Hence there exists $\left\{p_{1}\right\}$

$$
\text { such that } \lim \left(\sum_{j=1}^{\infty} x_{p_{i} p_{j}}\right)=0 \text { and } \frac{1 \neq m}{f_{1 \neq \infty}}\left(\sum_{i=1}^{\infty} x_{p_{i}} p_{j}\right)=0
$$

We now state and prove the theorem of Toeplitz.

THEOREM 1. Let $A=\left(a_{m, n}\right)$ be an infinte matrix. The necessary and sufficient conditions for $A$ to be regular are that the following three conditions must hold.

$$
\begin{aligned}
& \text { (a) } \operatorname{Sup}_{m}\left(\sum_{n=1}^{\infty}\left|a_{m, n}\right|\right)<\infty . \\
& \text { (b) } \lim _{m} a_{m, n}=0 \text { for each } n . \\
& \text { (c) } \underset{m}{\lim }\left(\sum_{n=1}^{\infty} a_{m, n}\right)=1 .
\end{aligned}
$$

PROOF. We now prove the sufficiency part of the theorem.

Let $A$ be an infinite matrix satisfying the conditions (a), (b) and (c). Let $\sigma=\left\{s_{n}\right\}$ be a sequence such that $s_{n} \rightarrow s$ as $n \rightarrow \infty$. Then $t_{m}$ converges for each $m$. We will now show that $t_{m} \rightarrow s$ as $m \rightarrow \infty$.

Given $\epsilon>0$, there is an integer $N$ such that $\left|s-s_{n}\right|\langle\epsilon \quad$ for $n>N$. By (c) there is an integer $M$ such that

$$
\left|1-\sum_{n=1}^{\infty} a_{m, n}\right|<\epsilon \text { for } m>M
$$

For $m>M$, using (a) we have

$$
\begin{aligned}
\left|s-t_{m}\right| & =\left|s-\sum_{n=1}^{\infty} a_{m, n} s_{n}\right| \\
& =\left|\sum_{n=1}^{\infty} a_{m, n}\left(s-s_{n}\right)+s\left(1-\sum_{n=1}^{\infty} a_{m, n}\right)\right| \\
& \left.\leq \sum_{n=1}^{N}+\underset{n=N+1}{\infty}\right)\left|a_{m, n}\right|\left|\left(s-s_{n}\right)\right|+|s| \epsilon \\
& \leq \sum_{n=1}^{N}\left|a_{m, n}\right|\left|\left(s-s_{n}\right)\right|+K \epsilon+|s| \epsilon .
\end{aligned}
$$


But by (b), there is an integer $M^{\prime}>M$ such that

$$
\left|a_{m, n}\right|<\epsilon / N
$$

for $M^{\prime}<m$. If $L=\sup _{n}\left(\left|s-s_{n}\right|\right)$, we have for $m>M^{\prime}$

$$
\left|s-t_{m}\right|<N L(\epsilon / N)+K \epsilon+|s| \epsilon \leq(L+K|s|) \epsilon
$$

(2.11) shows that $t_{m} \rightarrow s$ as $m \rightarrow \infty$.

We now prove the necessity part. We first set

$$
\begin{aligned}
& e_{n}^{(k)}=\left\{\begin{array}{lll}
1 & \text { if } n=k \\
0 & \text { if } n \neq k
\end{array}\right. \\
& e=\left\{e_{n}\right\} \text { whee for all } n, e_{n}=1 .
\end{aligned}
$$

$\left\{e_{n}^{(k)}\right\}$ and $e$ belong to (c). The necessity of (b) in Theorem 1 is obtained by noting that $A$ is convergence preserving and hence

$$
\underset{n}{1 \neq \infty}\left(e_{n}^{(k)}\right)=0=A\left(\lim _{n} \neq e_{n}^{(k)}\right)=\frac{1 i m}{m}\left(a_{m, k}\right)
$$

The necessity of condition (c) in Theorem 1 is obtained by observing that

$$
1=\lim _{n \rightarrow \infty}\left(e_{n}\right)=A\left(\lim _{n \rightarrow \infty} e_{n}\right)=\lim _{m \rightarrow \infty}\left(\sum_{n=1}^{\infty} a_{m, n}\right)
$$

Thus we need to demonstrate the validity of condition (a).

Suppose (a) does not hold. Then there exists a sequence of finite subsets $\left\{\sigma_{\mathrm{m}}\right\}$ of positive integers such that

$$
\max \left(\sigma_{m}\right)<\min \left(\sigma_{m+1}\right)
$$

for $m \in N$ (where $N$ is the set of positive integers) with the additional property

$$
\begin{array}{ll} 
& j \in \sigma_{m} a_{m, j}+\infty \\
\text { Let } & \alpha_{m}=\left.\left.\right|_{j} \in \sum_{\sigma_{m}} a_{m, j}\right|^{-1 / 2} \text { for } m \in N \\
\text { Let } & b_{m, n}=\alpha_{m}, \alpha_{n}\left(\sum_{j \sigma_{m}} a_{m, j}\right)
\end{array}
$$

We note that the columns and rows of the matrix $\left(b_{m, n}\right)$ converge to zero. Further it is not difficult to show that there is a submatrix $\left(b_{i_{m}, i_{n}}\right)$ of $\left(b_{m, n}\right)$ such that

$$
\underset{m \rightarrow m}{\lim }\left(\sum_{n \neq m}\left|b_{i_{m}}, i_{n}\right|\right)=0 \quad \text { (See [2], diagonal theorem). }
$$

We now define a sequence $\left[s_{n}\right]$ as follows. Let

$$
s_{n}=\left\{\begin{array}{l}
\beta_{n} \text { with } \sigma_{n}=u_{i_{k}} \text { if } n \quad \sigma_{i_{k}} \\
0 \text { if } n \notin U \sigma_{i_{k}}
\end{array}\right.
$$


Obviously $\left\{s_{n}\right\} \quad\left(c_{0}\right)$, the space of null sequences. Hence

$$
\sum_{n=1}^{\infty} b_{i_{m}, i_{n}}=\alpha_{i_{m}}\left(\sum_{j=1}^{\infty} a_{i_{m}, j} s_{j}\right)
$$

By the regularity of $A$ it is seen that

$$
\lim \left(\sum_{j=1}^{\infty} a_{i m}, j s_{j}\right) \text { exists }
$$

Hence $\quad \frac{1 i m}{m^{\ddagger} \infty}\left(\sum_{j=1}^{\infty} b_{i_{m}, i_{n}}\right)=0$.

Clearly we have

$$
\left|b_{i_{m}, i_{n}}\right|<\sum_{n \neq m}\left|b_{i_{m}}, i_{n}\right|+\left|\sum_{n=1}^{\infty} b_{i_{m}, i_{n}}\right|
$$

for $m \mathrm{~N}$.

We use $(2.17)$ and $(2.21)$ to obtain

$$
\underset{\mathrm{m} i m}{\lim }\left(\left|\mathrm{b}_{i_{m}, i_{n}}\right|\right)=0
$$

On the other hand $\underset{m}{1+m}\left(\left|b_{i_{m}}, i_{n}\right|=1\right.$.

The apparent contradiction in steps (2.23) and (2.24) shows that condition (a) must be valid. This now completes the proof of Theorem 1.

REMARK 1. The Diagonal theorem is mainly used to show the necessity of condition (a). The remaining parts of the proof are quite standard and we presented them for the sake of making the proof complete.

The authors would like to express their thanks to the referee for his suggestions that resulted in the present form of this paper. It is also possible to prove the non-archimedean analogue of Toeplitz's theorem, proved by Monna [3], who derived it by using the analogue of uniform boundedness principle over complete non-archimedean valued fields. As the proof is a trivial modification of the same techniques used in the classical case, this is left out.

\section{REFERENCES}

1. HARDY, G.H. Divergent Series, Oxford University Press, London, 1949.

2. ANTOSIK, P. A Diagonal Theorem for non-negative Matrices and Equicontinuous Sequences of mappings, Bull. Acad. Polon. Sci. Ser. Math. Astronom. Phys., 24 (1976), 855-859.

3. MONNA, A.F. Sur le Theoreme de Banach-Steinhauss, Proc. Kon. Ned. Akad. v. Wetensch., A 66, (1963), 121-131. 


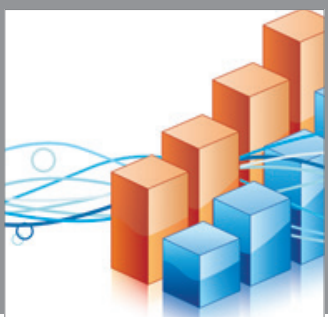

Advances in

Operations Research

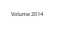

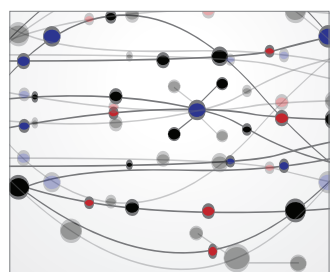

\section{The Scientific} World Journal
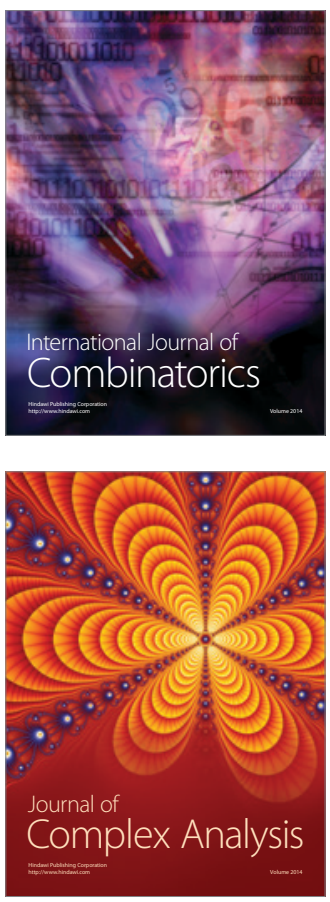

International Journal of

Mathematics and

Mathematical

Sciences
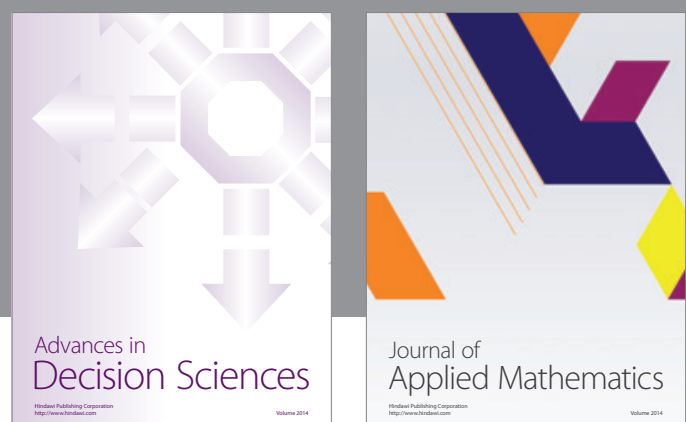

Journal of

Applied Mathematics
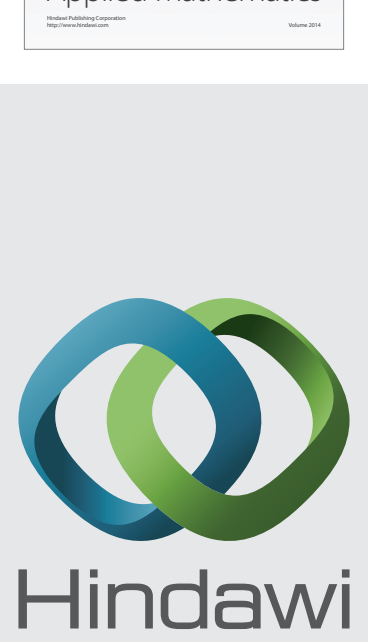

Submit your manuscripts at http://www.hindawi.com
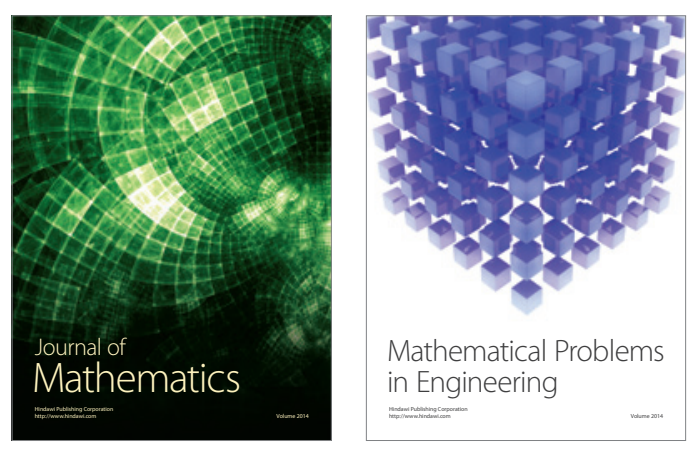

Mathematical Problems in Engineering
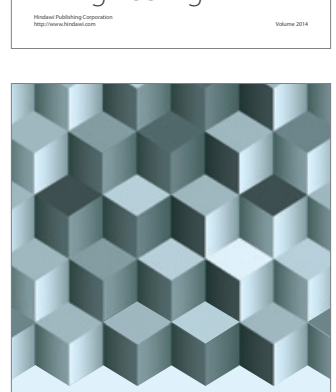

Journal of

Function Spaces
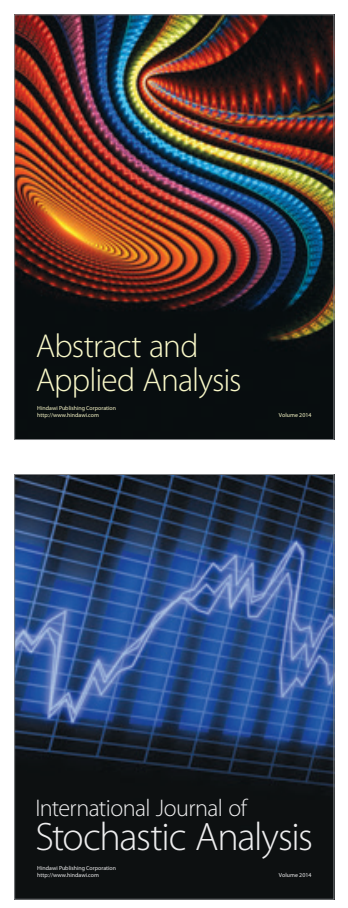

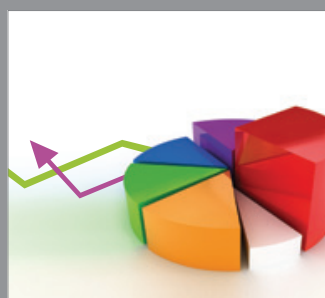

ournal of

Probability and Statistics

Promensencen
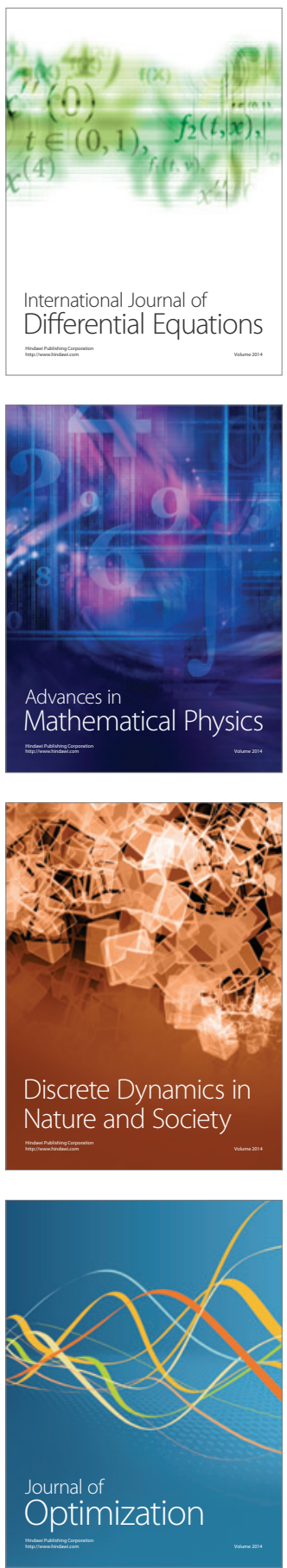